MS40-P05

\section{Crystallization of putative copper binding CrdA protein and helicase-like HP1026 protein from the human pathogen Helicobacter pylori}

Dubravka Matković-Čalogović1, Ivana Kekez ${ }^{1}$, Vigor Matković1, Mario Kekez ${ }^{1}$, Giuseppe Zanotti

1. Department of Chemistry, Faculty of Science, University of Zagreb, 10000 Zagreb, Croatia

2. Department of Biomedical Sciences, University of Padua, Padua, Italy

email: dubravka@chem.pmf.hr

Helicobacter pylori bacteria colonize the human stomach and can cause development of gastric diseases like atrophic gastritis, peptic ulcer, gastric adenocarcinoma and lymphoma of the mucosa-associated lymphoid tissue (MALT). ${ }^{1}$ For this purpose, proteins involved in survival of this bacterium have become an important target of research. Among them is the putative cooper binding CrdA protein and helicase-like HP1026 protein which were investigated in this work. CrdA is required for keeping the concentration of free copper ions below toxic levels ${ }^{2}$ while HP1026 has been classified as one of the heat shock proteins that are generally involved in regulation of the urease activity and adhesion to the epithelial cells. ${ }^{3}$

In order to reveal the structural and functional properties of these proteins, cloning, purification and crystallization trials (performed by the sitting and hanging drop vapor diffusion methods) have been carried out. Since crystallization of CrdA was not successful, the SUMO-fusion-CrdA protein incubacted with $\mathrm{Cu}^{2+}$ ions gave crystals, so far of poor diffraction quality. The optimization of crystallization is in progress. In the case of the HP1026 protein the thermal stability assay was also investigated. ATP-g-S and ADP strongly affected the protein stability by shifting the protein-complex denaturation to higher $t_{\mathrm{m}}$ values by $\mathrm{D}+3.52$ ${ }^{\circ} \mathrm{C}$ and $+5.18{ }^{\circ} \mathrm{C}$, respectively. This experiment together with the ATP hydrolysis assay (previously performed) was another confirmation that HP1026 belongs to the class of ATPase proteins. The results of this thermofluor assay were also applied in the crystallization experiments and crystallization of HP1026 supplemented with ADP resulted in hexagonal-shaped crystals. Diffraction data of HP1026 were measured at the XRD1 beamline (Elettra, Italy) but only to the resolution of $4 \AA$. The crystals belong to the hexagonal system $(a=116.22(2) \AA, c=238,38(2) \AA$, space group $\left.P 3_{1} 21\right)$. Since the sequence similarity of HP1026 and homologues with the known crystal structures is $30 \%$ or less, solving of structure of HP1026 was not successful so far. We are working on obtaining better quality crystals.

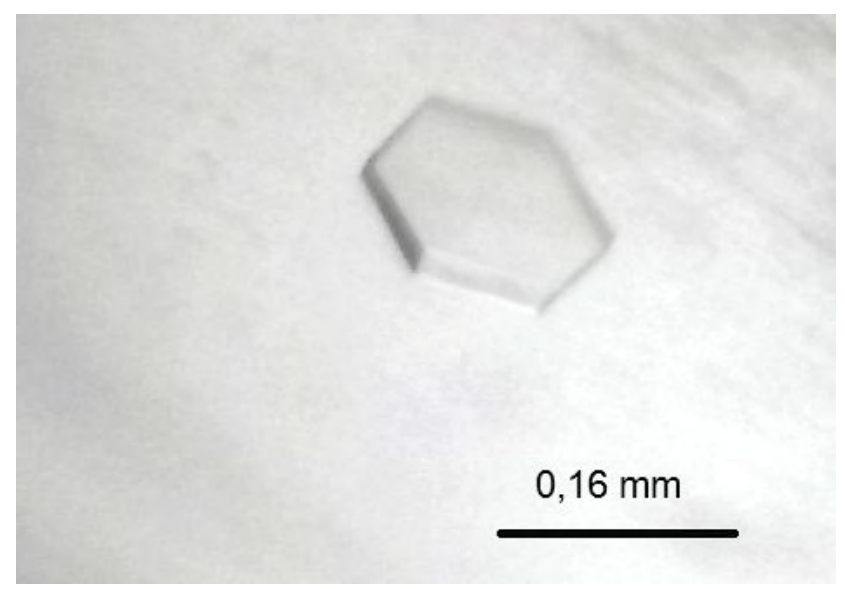

References:

[1] Rothenbacher, D. \& Brenner H. (2003). Microbes Infect. 5, 693-703.

[2] Waidner, B. et al. (2005). J. Bacteriol. 187, 4683-4688.

[3] Roncarati, D. et al. (2011). J. Bacteriol. 193, 5629-5636.

Keywords: crystallization, CrdA, HP1026 